AGráría

Revista Brasileira de Ciências Agrárias

v.3, n.3, p.283-288, jul.-set., 2008

Recife, PE, UFRPE, Www.agraria.ufrpe.br

Protocolo 362 - 27/03/2008 - Aprovado em 05/06/2008

José Imaña-Encinas ${ }^{1}$

Gustavo S. Ribeiro²

Lucélia A. de Macedo ${ }^{1}$

José E. de Paula ${ }^{3}$

Otacílio A. Santana ${ }^{1}$

${ }^{1}$ Departamento de Engenharia Florestal, Universidade de Brasília, DF, imana@unb.br; lucelia.macedo@ibama.gov.br; otaciliosantana@gmail.com

${ }^{2}$ Graduando em Engenharia Floresta//UNB, bolsista CNPq, Fone: (61) 3222-1009, CEP: 70.234-030, Brasília, DF, ribeiro_gustavo@hotmail.com; ${ }^{3}$ Departamento de Botânica, Universidade de Brasília, DF

\section{Composição arbórea de um trecho da floresta estacional semidecidual em Pirenópolis, Goiás}

\section{RESU M O}

A Floresta Estacional Semidecidual é um ecossistema bastante ameaçado no Brasil, cuja condução de levantamentos florísticos em florestas remanescentes é fundamental para fornecer dados que contribuam para a proposição de estratégias de manejo e a recuperação desses ecossistemas. Neste sentido, o objetivo do trabalho foi realizar o levantamento florístico de um trecho de Floresta Estacional Se-

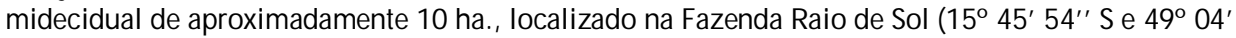
03" W) próxima à cidade de Pirenópolis, Goiás, inserida na biorregião do Ecomuseu do Cerrado. Para o levantamento do componente arbóreo foram alocadas dez parcelas de $20 \times 20 \mathrm{~m}$. Todos os indivíduos com DAP igual ou superior a $5 \mathrm{~cm}$ foram incluídos na amostragem, excluindo-se as lianas e os indivíduos mortos. Amostraram-se 742 indivíduos pertencentes a 83 espécies distribuídas em 67 gêneros e 36 famílias. As famílias de maior riqueza foram Fabaceae, Myrtaceae, Rubiaceae, Chrysobalanaceae e Apocynaceae, que contribuíram com $48 \%$ do total das espécies encontradas.

Palavras-chave: floresta seca tropical, florística, ecomuseu do cerrado, cerrado

\begin{abstract}
The Seasonal Semideciduous Forest is one of the most threatened Brazilian ecosystems. Therefore, to conduct forest mensuration studies in these areas is of vital importance to provide data for development of management strategies and they are also important for recovery of degraded areas. In view of this, the objective was to study the flora of a fragment of 10 ha of semideciduous forest at Raio de Sol farm (150 45' 54" S and 49 04' 03"' W) in Pirenópolis municipality, in the State of Goiás. It is inserted into the bioregion called Cerrado EcoM useum. Ten sample plots ( $20 \times 20 \mathrm{~m}$ ) were surveyed. In these plots all living trees with $5 \mathrm{~cm}$ diameter at $1.30 \mathrm{~m}$ above ground (DBH) were measured. 742 individuals belonging to 83 species, 67 genera, and 36 families were registered. The richest families in number of species were Fabaceae, M yrtaceae, Rubiaceae, Chrysobalanaceae and Apocynaceae, which contributed $48 \%$ of total species found.
\end{abstract}

Key words: tropical dry forest, floristic, cerrado ecomuseum, savannah 


\section{INTRODUÇÃO}

As florestas estacionais, também conhecidas como matas secas, incluem formações florestais caracterizadas por diversos níveis de caducifolia durante a estação seca; Nascimento et al. (2004) as denominam de matas secas de calcários, uma vez que ocorrem em áreas de afloramentos de calcário no bioma Cerrado. Na região Centro-Oeste essas florestas surgiram em solos particularmente propícios para a agricultura, razão pela qual foram tão devastadas que hoje é difícil imaginar que em várias regiões do Brasil Central esse tipo de floresta constituía uma densa cobertura vegetal dominante (Oliveira-Filho \& Ratter, 2001). Este tipo de comunidade vegetal não possui associação com cursos de água, revelando-se nos interflúvios em solos mais ricos em nutrientes (Nascimento et al., 2004). Além da atratividade conferida pela fertilidade do solo, os remanescentes dessas florestas concentram espécies arbóreas de significativo valor comercial, como Aspidosperma spp., Myracrodruon urundeuva, Cedrela fissilis, Tabebuia spp. (Felfili \& Silva Júnior, 2001) o que acaba aumentando a pressão antrópica sobre essas comunidades vegetais.

No estado de Goiás as perturbações antrópicas sobre os ecossistemas florestais causaram profundas alterações em sua cobertura vegetal, especificamente nas últimas três a quatro décadas tornando-se, hoje, premente a necessidade de se adotar medidas para reduzir o desmatamento dessa formação florestal (Galinkin, 2003). A concepção do Ecomuseu do Cerrado se enquadra plenamente no contexto dessas ações, uma vez que busca, desde 1997 (Instituto Huah do Planalto Central, 2003) conciliar o crescimento econômico com a conservação ambiental da região do divisor de águas das bacias hidrográficas do Alto Rio Corumbá.

A área geográfica do EcoMuseu do Cerrado abrange os municípios goianos de Pirenópolis, Corumbá, Cocalzinho, Abadiânia, Alexânia, Santo Antônio do Descoberto e Águas Lindas, ocupando uma área de $8.066 \mathrm{~km}^{2}$ (Nóbrega \& ImañaEncinas, 2006) com uma população aproximada de $240 \mathrm{mil}$ habitantes. Localiza-se entre os paralelos $15^{\circ} 21^{\prime}$ e $16^{\circ} 21^{\prime} \mathrm{S}$ e meridianos $48^{\circ} 04^{\prime}$ e $49^{\circ} 14^{\prime}$ W Gr. Os sete municípios que compõem a biorregião do EcoMuseu apresentam uma antropização de $58 \%$, sendo que as matas de galeria e as Florestas Estacionais foram as mais antropizadas (Nóbrega \& ImañaEncinas, 2006).

A condução de diversos levantamentos florísticos e de índole ecológica nessas comunidades vegetais é de importância fundamental para fornecer subsídios que permitam contribuir com o conhecimento e a preservação das áreas remanescentes.

O objetivo do presente estudo foi realizar o levantamento florístico do componente arbóreo de um trecho da Floresta Estacional Semidecidual, em uma área do município de Pirenópolis, Goiás.

\section{MATERIAL E MÉTODOS}

O remanescente florestal analisado se localiza na porção noroeste da área do EcoMuseu do Cerrado, no município de
Pirenópolis, Goiás. O levantamento florístico do componente arbóreo foi realizado em um trecho de aproximadamente 10 ha., situado a 15' 45' 54' S e 49 04' 03' 'W (SAD 69), com altitude próxima a 810 m, na Fazenda Raio de Sol.

A vegetação da área de estudo, classificada como Floresta Estacional Semidecidual fica contígua às áreas de florestas de galeria e de Cerrado sensu stricto. Os solos são profundos e bem drenados, provavelmente com baixa fertilidade natural e acidez acentuada. O clima do local, segundo a classificação climática de Köppen, é do tipo Aw, apresentando duas estações bem definidas: uma seca, no inverno, e uma úmida, no verão. A estação seca se prolonga até cinco meses, de maio a setembro, ocorrendo chuvas nos meses restantes, com precipitação média anual de $1.500 \mathrm{~mm}$. A temperatura média anual oscila entre $21,5^{\circ} \mathrm{C}$ e $24,9^{\circ} \mathrm{C}$ (Nóbrega \& Imaña-Encinas, 2006).

Ao longo da encosta do morro, no local de estudo, foram demarcadas e distanciadas em $80 \mathrm{~m}$, duas faixas compostas de cinco parcelas de 20 × 20 m cada uma, também distanciadas $80 \mathrm{~m}$; cada faixa totalizou $2.000 \mathrm{~m}^{2}$ de área de estudo, correspondendo a uma área amostral total de 0,4 ha.

Todos os indivíduos arbóreos, incluindo palmeiras, com DAP igual ou superior a $5 \mathrm{~cm}$ foram identificados e medidos com uma suta dendrométrica de $80 \mathrm{~cm}$. Foram excluídos da amostragem as lianas e os indivíduos mortos. A identificação das espécies foi realizada por um especialista em Dendrologia, in loco com base em caracteres dendrológicos das folhas e do fuste. Para alguns indivíduos se prepararam exsicatas para posterior confirmação no Herbário da Universidade de Brasília (UB), ficando as excicatas depositadas nesse herbário. Os indivíduos arbóreos foram identificados por espécies, gênero e família, pelo sistema APG II (Angiosperm Phylogeny Group II, 2003). Uma curva espécie-área foi plotada para avaliar a abrangência da amostragem.

\section{RESULTADOS E DISCUSSÃO}

Foram constatados, na área estudada, 742 indivíduos pertencentes a 83 espécies, distribuídos em 67 gêneros e 36 famílias (Tabela 1); quatro indivíduos arbóreos não puderam ser identificados.

Por meio da curva de suficiência amostral (Figura 1) foi possível demonstrar que a amostragem para a representação da variabilidade florística foi satisfatória, uma vez que se observa tendência à estabilidade (inflexão da curva) a partir da amostragem de uma área de $3.600 \mathrm{~m}^{2}$. A tendência linear indica a concordância com a curva de suficiência amostral, em que $3.600 \mathrm{~m}^{2}$ podem ser plenamente aceitos para interpretar corretamente a abrangência da amostragem; observa-se ainda que, a partir de $3.000 \mathrm{~m}^{2}$, se consolida um alto valor percentual $(80 \%)$ da ocorrência das correspondentes espécies arbóreas.

As famílias que apresentaram maior riqueza de espécies foram Fabaceae (11 espécies), Malvaceae (7 espécies), Myrtaceae (7 espécies), Rubiaceae (7 espécies), Chrysobalanaceae (4 espécies) e Apocynaceae (4 espécies). Essas seis famílias com 40 espécies contribuíram com 48,19\% do total das 
Tabela 1. Espécies arbóreas de um trecho de Mata Estacional Semidecidual na Fazenda Raio de Sol, Pirenópolis, e respectivos parâmetros de densidade

Table 1. Tree species of one fragment of the semideciduous seasonal forest at the Raio de Sol farm, Pirenópolis - Goiás state

\begin{tabular}{|c|c|c|c|c|}
\hline \multirow{2}{*}{ Espécie } & \multirow{2}{*}{ Família } & \multicolumn{2}{|c|}{ Densidade } & \multirow{2}{*}{ Caractesísticas } \\
\hline & & (n 04 ha $\left.^{-1}\right)$ & $\left(\mathrm{n} \mathrm{ha}^{-1}\right)$ & \\
\hline Agonandra brasiliensis Miers et Benth. \& Hook. f. & Opiliaceae & 5 & 12 & $\begin{array}{l}\text { Planta medicinal }(1,4) ; \text { Alimento da fauna (2, } \\
5)\end{array}$ \\
\hline Alibertia macrophylla K.Schum. & Rubiaceae & 22 & 55 & \\
\hline Alibertia vaccinioides K.Schum & Rubiaceae & 6 & 15 & \\
\hline Amaioua guianensis Aubl. & Rubiaceae & 30 & 75 & Alimento da fauna (2) \\
\hline Anadenanthera macrocarpa (Benth.) Brenan & Fabaceae & 10 & 25 & Produção de madeira (3) \\
\hline Apeiba tibourbou Aubl. & Malvaceae & 1 & 2 & Planta medicinal (5) \\
\hline Apuleia leiocarpa (Vogel) J. F. Macbr. & Fabaceae & 3 & 7 & Produção de madeira (3) \\
\hline Aspidosperma australe Müll. Arg. & Apocynaceae & 2 & 5 & Planta medicinal e corante $(2 ; 5)$ \\
\hline Aspidosperma discolor A. DC. & Apocynaceae & 4 & 10 & Planta medicinal (5) \\
\hline Aspidosperma subincanum Mart. & Apocynaceae & 20 & 50 & Produção de madeira $(3,5)$ \\
\hline Astronium fraxinifolium Schott & Anacardiaceae & 10 & 25 & Planta medicinal $(1,5)$; Alimento da fauna (5) \\
\hline Basiloxylon brasiliensis (Allemão) K. Schum. & Malvaceae & 2 & 5 & Produção de madeira (3) \\
\hline Bauhinia ungulata $L$. & Fabaceae & 8 & 20 & \\
\hline Bowdichia virgilioides H. B. \& Kunth & Fabaceae & 1 & 2 & Planta medicinal. (1); Planta melífera (4) \\
\hline Byrsonima crassifolia (L.) Kunth & Malpighiaceae & 1 & 2 & \\
\hline Byrsonima intermedia A. Juss. & Malpighiaceae & 22 & 55 & \\
\hline Callisthene major Mart. & Vochysiaceae & 23 & 57 & Planta medicinal (2) \\
\hline Calophyllum brasiliense Cambess & Plusiaceae & 1 & 2 & Alimento para a fauna (3) \\
\hline Cassia ferruginea (Schrader) Schrader ex DC. & Fabaceae & 1 & 2 & Produção de madeira (3) \\
\hline Cecropia pachystachya Trécul & Urticaceae & 2 & 5 & Alimento para a fauna (3) \\
\hline Cheiloclinium cognatum (Miers.) A.C. Smith & Celastraceae & 2 & 5 & Alimento para a fauna (5) \\
\hline Copaifera langsdorffii Desf. & Fabaceae & 6 & 15 & Planta medicinal (1) \\
\hline Coussarea contracta (Walp.) Müll. Arg. & Rubiaceae & 4 & 10 & \\
\hline Curatella americana L. & Dilleniaceae & 9 & 22 & Planta medicinal (1); Alimento para a fauna (3, \\
\hline Diospyros hispida A. DC. var. camporum Warm. & Ebenaceae & 5 & 12 & Alimento para a fauna e melífera (2) \\
\hline Diospyros hispida A. DC. var. hispida & Ebenaceae & 9 & 22 & Alimento para a fauna e melífera (2) \\
\hline Emmotum nitens (Benth.) Miers & Icacinaceae & 12 & 30 & Alimento para a fauna $(2,5)$ \\
\hline Eriotheca gracilipes (K. Schum.) A. Robyns & Malvaceae & 2 & 5 & Produção de pasta celulósica (3) \\
\hline Erythroxylum amplifolium Baill. & Erythroxylaceae & 1 & 2 & \\
\hline Erythroxylum daphnites Mart. & Erythroxylaceae & 14 & 35 & \\
\hline Eugenia bracteata Rich. & Myrtaceae & 3 & 7 & \\
\hline Eugenia gamaeana Glaz. & Myrtaceae & 6 & 15 & \\
\hline Euplassa inaequalis (Pohl) Engl. & Proteaceae & 5 & 12 & Alimento para a fauna (5) \\
\hline Ficus gardneriana (Miq.) Miq. & Moraceae & 2 & 5 & Produção de lâminas (6) \\
\hline Gomidesia pubescens (DC.) D. Legrand & Myrtaceae & 1 & 2 & \\
\hline Gomidesia schaueriana O. Berg. & Myrtaceae & 4 & 10 & \\
\hline Guazuma ulmifolia Lam. & Malvaceae & 1 & 2 & Alimento para fauna (3); Planta medicinal (5) \\
\hline Guettarda pohliana Müll. Arg. & Rubiaceae & 17 & 42 & \\
\hline Guettarda viburnoides Cham. \& Schltdl. & Rubiaceae & 25 & 62 & Alimento para a fauna (2) \\
\hline Hancornia pubescens Nees \& C. Mart. & Apocynaceae & 3 & 7 & Alimento para a fauna e planta medicinal (5) \\
\hline Hirtella glandulosa Spreng. & Chrysobalanaceae & 3 & 7 & Alimento para a fauna (2) \\
\hline Hymenaea courbaril L. & Fabaceae & 3 & 7 & Alimento para a fauna (3) \\
\hline Inga sessilis (Vell.) Mart. & Fabaceae & 1 & 2 & alimento para a fauna (2) \\
\hline Kielmeyera coriacea Mart. \& Zucc. var. coriaceae & Plusiaceae & 1 & 2 & Planta medicinal (1) \\
\hline Laplacea fruticosa (Schrad.) Kobuski & Theaceae & 1 & 2 & Ornamental e recuperação de áreas (5) \\
\hline Licania apetala (E. Mey.) Fritsch & Chrysobalanaceae & 21 & 52 & Produção de madeira (6) \\
\hline Licania nitida Hook. F. & Chrysobalanaceae & 4 & 10 & Produção de madeira (6) \\
\hline Licania octandra (Hoff. ex Roem. \& Shult.) Kuntze & Chrysobalanaceae & 27 & 67 & Produção de madeira (6) \\
\hline Luehea divaricata Mart. \& Zucc. & Malvaceae & 18 & 45 & Planta medicinal e melífera (5) \\
\hline Matayba guianensis Aubl. & Sapindaceae & 2 & 5 & Melífera e alimento para a fauna (5) \\
\hline Miconia calvescens Schrank \& Mart. ex DC. & Melastomataceae & 2 & 5 & \\
\hline Myracrodruon urundeuva M. Allemão & Anacardiaceae & 1 & 2 & Planta medicinal $(1,5)$; Alimento para a fauna \\
\hline Myrcia gamaeana Glaz. & Myrtaceae & 2 & 5 & \\
\hline Myrcia multiflora (Lam.) DC. & Myrtaceae & 20 & 50 & \\
\hline
\end{tabular}


Continuação...

\begin{tabular}{|c|c|c|c|c|}
\hline Myrcia tomentosa (Aubl.) DC. & Myrtaceae & 6 & 15 & Alimento para a fauna (2) \\
\hline Oenocarpus distichus Mart. & Aracaceae & 5 & 12 & \\
\hline Ouratea schomburgkii (Planch.) Engl. & Ochnaceae & 2 & 5 & \\
\hline Palicourea gardneriana (Müll. Arg.) Standl. & Rubiaceae & 2 & 5 & \\
\hline Pera glabrata (Schott.) Poepp. ex Baill. & Euphorbiaceae & 5 & 12 & Alimento para a fauna $(3,5)$ \\
\hline Pouteria gardneriana (A. DC.) Radllk. & Sapotaceae & 12 & 30 & \\
\hline Protium brasiliense (Spreng.) Engl. & Burseraceae & 4 & 10 & \\
\hline Protium heptaphyllum (Aubl.) Marchand & Burseraceae & 88 & 220 & Alimento para a fauna (3) \\
\hline $\begin{array}{l}\text { Pseudobombax tomentosum (Mart. \& Zucc.) } \\
\text { Robyns }\end{array}$ & Malvaceae & 2 & 5 & Ornamental e confecção de tecidos $(2,4)$ \\
\hline Pterodon emarginatus Vogel & Fabaceae & 1 & 2 & Planta medicinal e melífera (5) \\
\hline Qualea dichotoma (Mart.) Warm. & Vochysiaceae & 16 & 40 & Ornamental e produção de POM (3) \\
\hline Roupala montana Aubl. & Proteaceae & 8 & 20 & Usada na produção de POM e melífera (4) \\
\hline Salacia amygdalina Peyr. & Celastraceae & 15 & 37 & \\
\hline Sclerolobium paniculatum Vogel & Fabaceae & 11 & 27 & Melífera, usada na recuperação de áreas $(2,4)$ \\
\hline Securidaca retusa Benth. & Polygalaceae & 5 & 12 & \\
\hline Siparuna guianensis Aubl. & Monimiaceae & 9 & 22 & \\
\hline Sorocea guilleminiana Gaudich. & Moraceae & 3 & 7 & \\
\hline Sterculia chicha A. St.-Hil. ex Turpin & Malvaceae & 6 & 15 & Pasta celulósica (3) \\
\hline Stryphnodendron adstringens (Mart.) Covillei. & Fabaceae & 2 & 5 & Planta medicinal. (1) \\
\hline Symplocos frondosa Brand & Symplocaceae & 1 & 2 & \\
\hline Tabebuia ochracea (Cham.) Standl. & Bignoniaceae & 15 & 37 & Planta medicinal (4) \\
\hline Tapirira guianensis Aubl. & Anacardiaceae & 62 & 155 & Alimento para a fauna $(3,5)$ \\
\hline Tapura amazonica Poepp. & Dichapetalaceae & 11 & 27 & Alimento para a fauna (5) \\
\hline Terminalia argentea Mart. & Combretaceae & 4 & 10 & Planta medicinal (5) \\
\hline Terminalia glabrescens Mart. & Combretaceae & 1 & 2 & Arborização (5) \\
\hline Terminalia phaeocarpa Eichler & Combretaceae & 2 & 5 & Melífera (5) \\
\hline Triplaris gardneriana Wedd. & Polygonaceae & 1 & 2 & Planta medicinal (2); Alimento para a fauna (5) \\
\hline Virola sebifera Aubl. & Myristicaceae & 15 & 37 & Alimento para a fauna $(3,5)$; \\
\hline Xylopia aromatica (Lam.) Mart. & Annonaceae & 13 & 32 & Planta medicinal (1); Alimento para a fauna $(4,5)$ \\
\hline Indivíduos não identificados & & 4 & & \\
\hline Total & & 742 & 1.855 & \\
\hline
\end{tabular}

(1) Silva et al, 2001; (2) Lorenzi, 2002a; (3) Lorenzi, 2002b; (4) Silva Jr., 2005; (5) IBGE, 2002; (6) IPT, 2008

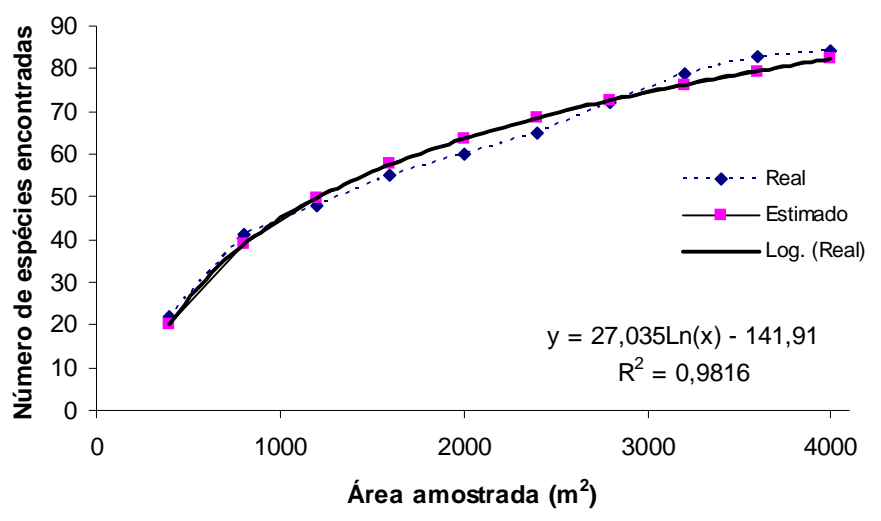

Figura 1. Curva de suficiência amostral de um trecho da Floresta Estacional Semidecidual na Fazenda Raio de Sol, Pirenópolis, Goiás

Figure 1. Efficiency-sample curve for a fragment of the semideciduous seasonal forest at the Raio de Sol farm, Pirenópolis, in the State of Goiás

espécies amostradas e dezenove famílias (23\%) foram representadas por uma só espécie.

Lopes et al. (2002), Paula et al. (2002), Silva et al. (2004) e Nascimento et al. (2004) estudando Matas Estacionais Semideciduais apontaram a Leguminosae como a família de maior riqueza. Pela classificação APG II (Aspidosperma Phylogeny Group, 2003) a família Leguminosae, neste trabalho, passou a ser considerada Fabaceae. Silva et al. (2004) comentaram que a capacidade de fixar nitrogênio apresentada por espécies desta família poderá ser considerada uma boa estratégia de vida quando os solos que sustentam essas florestas em topos e encostas de morros apresentarem baixa fertilidade natural, como é o caso da área estudada. A família Leguminosae é extremamente importante na estrutura da vegetação de florestas e do cerrado, sendo imprescindível à sua preservação na dinâmica populacional destas comunidades (Fidelis \& Godoy, 2003).

Em uma Floresta Estacional Semidecidual, em Ibituruna, MG, Silva et al. (2003) descreveram uma considerável riqueza florística, 191 espécies arbóreas com diâmetro acima de $5 \mathrm{~cm}$, sendo Fabaceae, Myrtaceae e Lauraceae as famílias mais ricas, para uma área amostral de 1,04 ha. Em estudo realizado em Floresta Estacional Semidecídua em Minas Gerais, Lopes et al. (2002) também constataram uma riqueza de 121 espécies com indivíduos acima de $4,8 \mathrm{~cm}$ de diâmetro, sendo a Leguminosae a família de maior riqueza, seguida de Lauraceae, Rubiaceae e Flacourtiacee, em uma área amostral de 1 ha. No presente trabalho foram encontradas 83 espécies, correspondendo a 43 e $68 \%$, respectivamente, dos trabalhos acima indicados. Pode-se deduzir que, provavelmente, na área estudada ocorreu ação antrópica com corte seletivo de algumas espécies. 
Nascimento et al. (2004) encontraram 21 famílias em uma área amostrada em um remanescente de floresta estacional decidual de encosta em Monte Alegre, GO; essas 21 famílias estavam representadas por 52 espécies arbóreas com DAP maio a $5 \mathrm{~cm}$ em uma proporção de espécies por gênero na família Bignoniaceae de 2:1 e Leguminosae de 1,41:1, como sendo os maiores valores levantados. Em relação ao presente trabalho, a proporção encontrada foi de 2:1, duas espécies por família.

No presente estudo as famílias Apocynaceae, Burseraceae, Chrysobalanaceae, Combretaceae, Ebenaceae, Erythroxylaceae, Malpighiaceae, Myrtaceae e Rubiaceae apresentaram mais de uma espécie por gênero (Tabela 1). Os gêneros mais ricos em número de espécies foram Aspidosperma, Licania, Terminalia e Myrcia, apresentando três espécies cada um.

As espécies Protium heptaphyllum, Tapirira guianensis, Amaioua guianensis, Licania octandra e Guettarda virbunoides se destacaram pela sua densidade, visto que acumularam mais de $30 \%$ do total de indivíduos.

Dentre as espécies encontradas pode-se citar algumas de ocorrência comum em florestas de galeria: Tapirira guianensis, Protium heptaphyllum, Callisthene major e Amaioua guianensis, o que confirma a influência desse ecossistema no trecho vegetal arbóreo estudado. Estudos realizados em florestas de galeria do Distrito Federal apontaram essas espécies entre as principais (Sampaio et al., 1997; Sevilha et al., 2001).

Encontrou-se, em algumas parcelas do trecho florestal estudado, um número elevado de indivíduos jovens de Virola sebifera mas nenhum indivíduo adulto. Indivíduos adultos desta espécie foram encontrados predominantemente próximos às parcelas situadas nos pontos mais altos da encosta.

Dentre as espécies de valor comercial que ocorrem na área de estudo se destaca Astronium fraxinifolium, espécie declarada oficialmente ameaçada de extinção pela portaria $\mathrm{n}^{\circ}$. 37-N de 03/04/1992 do IBAMA.

Algumas espécies encontradas na área de estudo, como Alibertia macrophylla, Calophyllum brasiliense, Cheiloclinium cognatum, Emmotum nitens, Eugenia bracteata, Gomidesia regneliana, Protium brasiliense, Siparuna guianensi e Virola sebifera, servem de fonte de alimento para a fauna silvestre (Tabela 1), em razão da produção de sementes e frutos apreciados por vários representantes dessa fauna e, em conseqüência, devem ser amplamente preservadas.

\section{CONCLUSÕES}

Na floresta estacional semidecidual estudada em um trecho de 0,4 ha, foram encontrados 742 indivíduos arbóreos, distribuídos em 83 espécies, mostrando ampla riqueza florística, que deve ser conservada.

O trecho estudado apresenta espécies de importância comercial, inclusive ameaçadas de extinção, como Astronium fraxinifolium, além de espécies que produzem frutos e sementes para a dieta de espécies da fauna, o que eleva o seu valor a essa comunidade vegetal.

\section{LITERATURA CITADA}

Angiosperm Phylogeny Group. An update of the Angiosperm Phylogeny Group classification for the orders and families of flowering plants: APG II. Botany Journal Linnean Society. v.141, n.4, p.399-436, 2003.

Felfilli, J. M.; Silva Júnior, M. C. Biogeografia do bioma cerrado: estudo fitofisionômico na chapada do Espigão Mestre do São Francisco. Brasília: Universidade de Brasília, Departamento de Engenharia Florestal, 2001.152p.

Fidelis, A. T.; Godoy, S. A. P. Estrutura de um cerrado stricto sen$s u$ na gleba cerrado pé de gigante, Santa Rita do Passa Quatro, SP. Acta Botanica Brasílica, v.17, n.4, p.531-539, 2003.

Galinkin, M. (ed.). Geogoiás 2002. Goiânia: Agência Ambiental de Goiás, 2003. 239p.

IBGE - Instituto Brasileiro de Geografia e Estatística. Árvores do Brasil Central: espécies da região geoeconômica de Brasília. Rio de Janeiro: Diretoria de Geociências, 2002. 417p.

Instituto Huah do Planalto Central. Almanaque Ecomuseu do Cerrado. Brasília: Ministério do Meio Ambiente, 2003. 44 p.

IPT - Instituto de Pesquisas Tecnológicas. Recursos florestais. Madeiras e produtos derivados. http://www.ipt.br/areas/ctfloresta/lmpd/madeiras/busca. 09 Mar. 2008.

Lopes, W. P.; Paula, A.; Sevilha, A. C.; Silva, A. F. Composição da flora arbórea de um trecho de floresta estacional no jardim botânico da Universidade Federal de Viçosa (face sudoeste), Viçosa, Minas Gerais. Revista Árvore, v.26, n.3, p.339-347, 2002.

Lorenzi, H. Árvores brasileiras: manual de identificação e cultivo de plantas arbóreas do Brasil. v.1. Nova Odessa, São Paulo: Plantarum, 2002a.368p.

Lorenzi, H. Árvores brasileiras: manual de identificação e cultivo de plantas arbóreas do Brasil. v.2. Nova Odessa, São Paulo: Plantarum, 2002b. 368p.

Nascimento, A. R. T.; Felfili, J. M.; Meirelles, E. M. Florística e estrutura da comunidade arbórea de um remanescente de floresta estacional decidual de encosta, município de Monte Alegre, GO, Brasil. Acta Botanica Brasílica, v.18, n.3, p.663-674, 2004.

Nobrega, R. C. da; Imaña-Encinas, J. Uso do solo do projeto Ecomuseu do Cerrado. Revista Árvore, v.30, n.1, p.117-122, 2006.

Oliveira-Filho, A. T.; Ratter, J. A. Padrões florísticos das matas ciliares da região do cerrado e a evolução das paisagens do Brasil Central durante o quaternário tardio. In: Rodrigues, R. R.; Leitão Filho, H. (eds.). Matas Ciliares: Conservação e recuperação. São Paulo: USP, FAPESP, 2001. p.73-89.

Paula, A.; Silva, A. F.; Souza, A. L.; Santos, F. A. M. Alterações florísticas ocorridas num período de quatorze anos na vegetação arbórea de uma floresta estacional semidecidual em Viçosa-MG. Revista Árvore, v.26, n.6, p.743-749, 2002.

Sampaio, A. B.; Nunes, R. V.; Walter, B. M. T. Fitossociologia de uma mata de galeria na fazenda sucupira do Cenargen, Brasília, DF. In: Leite, L. L.; Saito, H. S. (ed.). Contribuição ao conhecimento ecológico do Cerrado. Brasília: Universidade de Brasília, Departamento de Ecologia, 1997. 326p.

Sevilha, A. C.; Paula, A.; Lopes, W. P.; Silva, A. F. Fitossociologia do estrato arbóreo de um trecho de floresta estacional no Jardim Botânico da Universidade Federal de Viçosa (fase sudoeste). Revista Árvore, v.25, n.4, p.431-443, 2001. 
Silva Júnior, M. C. 100 Árvores do cerrado: guia de campo. Brasília: Rede de Sementes do Cerrado, 2005. 278p.

Silva, N. R. S.; Martins, S. V.; Meira Neto, J. A. A.; Souza, A. L. Composição florística e estrutura de uma floresta estacional semidecidual montana em Viçosa, MG. Revista Árvore, v.28, n.3, p.397-405, 2004.
Silva, S. R. et al. Guia de plantas do cerrado utilizado na Chapada dos Veadeiros. Brasília: WWF, 2001. 132p.

Silva, V. F.; Venturini, N.; Oliveira-Filho, A. T.; Macedo, R. L. G.; Carvalho, W. A. C.; Van den Berg, E. Caracterização estrutural de um fragmento de floresta semidecídua no município de Ibituruna, MG. Cerne, v.9, n.1, p.92-106, 2003. 